\section{Sobrevida e fatores de risco para mortalidade neonatal em uma coorte de nascidos vivos de muito baixo peso ao nascer, na Região Sul do Município de São Paulo, Brasil}

\author{
Survival and risk factors for neonatal mortality in \\ a cohort of very low birth weight infants in the \\ southern region of São Paulo city, Brazil
}

\author{
1 Faculdade de Saúde Pública, \\ Universidade de São Paulo, \\ São Paulo, Brasil. \\ 2 Faculdade de Medicina, \\ Universidade de São Paulo, \\ São Paulo, Brasil. \\ ${ }^{3}$ Department of Epidemiology \\ and Reproductive Health, \\ London School of Hygiene \\ and Tropical Medicine \\ London, U.K. \\ Correspondência \\ M. F. Almeida \\ Departamento de \\ Epidemiologia, Faculdade de \\ Saúde Pública, Universidade \\ de São Paulo. \\ Av. Dr. Arnaldo 715, São Paulo, \\ SP 01246-904, Brasil. \\ marfural@usp.br
}

\begin{abstract}
Population studies can help identify the complex set of risk factors for neonatal mortality among very low birth weight infants. A cohort (20002001) of 213 live newborns with birth weight < 1,500g in the southern region of São Paulo city, Brazil, was studied (112 neonatal deaths and 101 survivors). Data were obtained from home interviews and hospital records. Survival analysis and multiple Cox regression were performed. The high mortality in the delivery room and in the first day of life among neonates $<1,000 \mathrm{~g}$ and $<28$ weeks gestational age and the absence of survival in neonates $<700 \mathrm{~g}$ suggest that care was actively oriented towards newborns with better prognosis. Increased risk of neonatal mortality was associated with maternal residence in slum areas, history of previous cesarean(s), history of induced abortion(s), adolescent motherhood, vaginal bleeding, and lack of prenatal care. Cesarean section and referral of the newborn to the hospital nursery showed protective effects. Birth weight less than 1,000g and Apgar index $<7$ were associated with increased risk. The high mortality was due to poor living conditions and to maternal and neonatal characteristics. Improvement in prenatal and neonatal care could reduce neonatal mortality in these infants.
\end{abstract}

Low Birth Weight Infant; Neonatal Mortality; Cohort Studies; Survival Analysis

\author{
Marcia Furquim de Almeida 1 \\ Gizelton Pereira Alencar 1 \\ Daniela Schoeps 1 \\ Hillegonda Maria Dutilh Novaes 2 \\ Oona Campbell 3 \\ Laura C. Rodrigues 3
}

\section{Introdução}

O Brasil, assim como outros países em desenvolvimento, vem apresentando tendência de declínio da mortalidade infantil, e a mortalidade neonatal passou a ser seu principal componente (DATASUS. http://tabnet.datasus.gov. br/cgi/idb2008/c0102b.htm, acessado em 14/ Set/2010) 1. Apesar de os nascimentos de muito baixo peso representarem apenas uma pequena parcela do total, estes concentram grande parte dos óbitos neonatais, o que destaca a importância de se conhecerem características dos subgrupos de peso e idade gestacional, bem como seus fatores de risco.

$\mathrm{O}$ acesso aos serviços de saúde e a qualidade destes desempenham importante papel na sobrevivência de recém-nascidos de muito baixo peso ao nascer 2,3,4. Grande parte dos estudos sobre a mortalidade destes neonatos foi realizada em hospitais de referência 2,3,4,5,6,7,8,9; entretanto, pesquisas realizadas em hospitais terciários podem homogeneizar as características da população usuária, devido ao seu acesso diferenciado.

Estudos populacionais podem trazer contribuições para a compreensão da mortalidade neonatal de nascimentos de muito baixo peso, pois são capazes de apreender a heterogeneidade das condições de vida, das características maternas e da gestação, além de diferenças de acesso à assistência pré-natal, ao parto e ao recém-nascido, existentes em grandes metrópoles. Tal heteroge- 
neidade, porém, nem sempre é possível de ser captada em estudos de coorte obtida em hospitais terciários de referência.

Foi realizado um estudo de base populacional para analisar a sobrevida e os fatores de risco possivelmente associados à mortalidade neonatal em uma coorte de recém-nascidos de muito baixo peso, na Região Sul do Município de São Paulo, em 2000/2001.

\section{Métodos}

A Região Sul do Município de São Paulo é formada por 14 distritos administrativos. Em 2000/2001, residiam nessa área 2.465.800 habitantes, representando $23,4 \%$ da população. Foi obtida uma coorte retrospectiva de 312 nascidos vivos hospitalares com menos de 1.500g (145 óbitos neonatais e 167 sobreviventes) de mães residentes na região, por meio da linkage de declarações de nascidos vivos e de óbitos neonatais (01/Ago/2000 a 31/Jan/2001). No segundo semestre de 2001, foram realizadas entrevistas domiciliares e aplicado protocolo para obter dados dos prontuários hospitalares. Os nascimentos e óbitos neonatais se distribuíram em 37 hospitais do município. As perdas mais elevadas deveram-se à não localização de endereços para $20,3 \%$ (34/167) dos sobreviventes e para $13,1 \%$ (19/145) dos óbitos neonatais. As recusas para entrevistas domiciliares e a não localização dos prontuários nos hospitais foi maior entre os sobreviventes - 24\% (32/133) - que entre os óbitos neonatais $-12,5 \%$ (14/112). O tempo de acompanhamento correspondeu a 27 dias, e a coorte final foi composta por 213 nascidos vivos (112 óbitos neonatais e 101 sobreviventes).

Empregou-se a definição da Classificação Internacional de Doenças (CID), que considera nascido vivo todo concepto que apresenta sinais vitais ao nascer 10 . A idade ao morrer foi mensurada em dias. Para óbitos ocorridos em menos de 24 horas, utilizou-se o valor 0,5 dia. A idade gestacional foi obtida por meio de algoritmo privilegiando os dados hospitalares: (1) dados de ultrassonografia; (2) idade gestacional registrada em semanas obtida por diferentes métodos de avaliação; (3) data da última menstruação registrada no prontuário e (4) data da última menstruação obtida na entrevista domiciliar.

As variáveis foram agrupadas em sete blocos: - B1 - Características socioeconômicas das mães: renda per capita em salários mínimos; local de residência; etnia autorreferida; escolaridade da mãe.

- B2 - História reprodutiva materna (gestações anteriores): número de filhos; presença de perda fetal; cesárea e aborto provocado anteriores; intervalo interpartal.

- B3 - Características psicossociais maternas: estado marital no momento do nascimento; percepção do estado de saúde pré-gestacional autorreferida; planejamento da gravidez; reações da mãe e da família à gravidez; violência doméstica durante a gestação, avaliada pelo Abuse Assessment Screning 11; mãe adolescente $(<20$ anos).

- B4 - Condições da gestação atual: IMC (índice de massa corpórea); presença de diabetes e doença renal crônica durante a gestação (autorreferidas); sangramento; hospitalização durante a gestação; presença de doença hipertensiva da gravidez; tipo de gravidez; fumo durante a gestação. Assistência pré-natal foi obtida nas entrevistas e foi considerada adequada, quando foi feita a primeira consulta no primeiro trimestre da gestação, com pelo menos uma medida de pressão arterial materna, ausculta do coração do feto e realização de exames (urina e sangue) pelo menos uma vez. Foi considerada não adequada quando essas condições estavam ausentes e não foi feito pré-natal 12 e uso de corticoesteroide (protocolo hospitalar).

- B5 - Características do parto: parto realizado no primeiro serviço de saúde; presença de placenta prévia; tocotraumatismo; ruptura prévia de membranas, início prematuro de trabalho de parto e tipo de parto, que foram obtidos dos prontuários e porte do hospital 13.

- B6 - Condições do recém-nascido: peso ao nascer; idade gestacional; sexo; índice de Apgar e presença de anomalias congênitas, obtidos dos prontuários hospitalares. Foram considerados como tendo retardo de crescimento intrauterino (RCIU) os recém-nascidos com peso ao nascer inferior ao percentil 10, segundo idade gestacional 14 .

- B7 - Cuidados ao recém-nascido: avaliação do tipo de atenção prestada ao recém-nascido, como uso de surfactante; berçário normal somente; berçário semi-intensivo; UTIN (Unidade de Terapia Intensiva Neonatal) - inclui os recém-nascidos que receberam este cuidado (após o parto e/ou posterior) - e transferência de hospital.

Foi calculada a incidência de óbitos $(\lambda)$ durante o período de observação ${ }^{15}$. O modelo de regressão de Cox foi empregado para avaliar o efeito de covariáveis sobre a força de mortalidade. Inicialmente, realizou-se regressão simples para a pré-seleção de variáveis $(\mathrm{p}<0,20)$ de cada bloco. Posteriormente, foi empregada análise hierarquizada 16, na qual a regressão múltipla foi realizada com as variáveis pré-selecionadas dos Blocos 1 e 2, sendo adicionadas, a seguir, as variáveis pré-selecionadas de cada bloco subse- 
quente. Para o modelo final, considerou-se $\mathrm{p}<$ 0,05 . Foram cumpridos os princípios contidos na Declaração de Helsinki, além de o estudo ter sido aprovado pelo Comitê de Ética em Pesquisa da Faculdade de Saúde Pública da Universidade São Paulo.

\section{Resultados}

As perdas foram elevadas e superestimaram em $15 \%$ a probabilidade de morte $(52,6 \%)$, mas a média do peso ao nascer das perdas $(1.213,9 \mathrm{~g})$ e da população de estudo $(1.027,7 \mathrm{~g})$ não foi estatisticamente significante $(\mathrm{p}=0,2649)$. Metade dos recém-nascidos morreu antes de completar 12 dias de vida. No primeiro dia de vida, ocorreram 51 óbitos e, destes, 14 se deram na sala de parto. Entre os que morreram nas primeiras 12 horas de vida, $86 \%$ tinham peso inferior a $1.000 \mathrm{~g}$. A mortalidade permaneceu elevada até o quarto dia de vida, reduzindo lentamente a partir do quinto dia (Figuras 1 e 2).

Os recém-nascidos foram agrupados em peso inferior a 1.000g (extremo baixo peso - EBPN) e 1.000g-1.499g. Nos dois grupos, os óbitos se concentraram nos primeiros dias de vida (Figura 2). Porém, nos EBPN, a probabilidade de morte no primeiro dia de vida foi 5,5 vezes a do grupo 1.000g-1.499g (Figura 1). Não houve sobreviventes com peso inferior a $700 \mathrm{~g}$.

O risco de morte no grupo EBPN era 4,8 vezes daquele no grupo de 1.000g-1.499g (IC95\%: 3,17,2; $\mathrm{p}<0,001)$. As estimativas de incidência de óbitos $(\lambda)$ indicam a velocidade de 0,104 criança/dia para o grupo abaixo de $1.000 \mathrm{~g}$ e de 0,013 criança/dia para os de 1.000 g e mais. A razão de incidências de óbitos foi de 7,8 (IC95\%: 5,2-12,1; $\mathrm{p}<0,01)$.

Foi adotada a definição de nascidos vivos da CID-10, sendo incluídos na coorte seis nascidos vivos com idade gestacional abaixo de 22 semanas. Outros seis recém-nascidos da coorte tinham idade gestacional acima de 36 semanas; todos apresentavam RCIU, resultado que se deveu possivelmente ao fato de a idade gestacional ter se baseado em diferentes formas de estimação.

Os recém-nascidos foram separados em dois grupos: idade gestacional abaixo de 28 semanas (prematuros extremos) e igual a ou acima de 28 semanas (Figura 2). Nos dois grupos, a probabilidade de morte foi elevada no primeiro dia de vida, e quase $40 \%$ dos recém-nascidos prematuros extremos morreram antes de completar 24 horas de vida. Até o quarto dia de vida, já havia morrido mais da metade dos recém-nascidos com idade gestacional abaixo de 28 semanas, os quais tinham mais que o dobro de chance de ocorrência de óbito que os de 28 semanas ou mais ( $\mathrm{HR}=2,8$; IC95\%: 1,9-4,1; $<<0,01)$. A estimativa da incidência de óbitos $(\lambda)$ é de $0,077 /$ criança/dia para os recém-nascidos de menos de 28 semanas, e de 0,010 óbitos/dia para os de 28 semanas ou mais. A intensidade de ocorrência de óbito dos recémnascidos extremante prematuros é quase quatro vezes (razão de incidência de óbitos = 3,9; IC95\%: $2,7-5,8$; $p<0,01$ ) maior que entre os de 28 semanas ou mais. A interação entre as variáveis peso ao nascer e idade gestacional foi testada, porém não se mostrou estatisticamente significante (HR = 1,5; IC95\%: 0,6-3,6; $\mathrm{p}=0,388$ ).

Com relação às principais características da atenção ao parto e ao recém-nascido, verificouse que a proporção de mães que não realizou o parto no primeiro serviço de saúde procurado por falta de vagas foi de $15,5 \%$; três recém-nascidos foram transferidos de hospital, 30 (14,1\%) foram para berçário normal, 36 (16,9\%) para o berçário semi-intensivo e $144(67,6 \%)$ foram encaminhados para a UTIN. O surfactante foi utilizado em 52,0\% dos recém-nascidos; nos nascidos com menos de $1.000 \mathrm{~g}(18,8 \%)$, seu uso foi pequeno.

Foram excluídas as variáveis não associadas ao desfecho ( $\mathrm{p}>0,20)$ do Bloco 1: renda per capita familiar, etnia e escolaridade; Bloco 2: número de filhos; Bloco 3: tipo/tempo de união, planejamento da gestação e reações das mães e das famílias; Bloco 4: IMC materno, tipo de gravidez e hábito de fumar na gestação; Bloco 5: placenta prévia, ruptura prematura de membrana e início de trabalho parto prematuro; parto no primeiro serviço de saúde; Bloco 6: RCIU; Bloco 7: utilização de berçário semi-intensivo/observação.

Foi realizada uma regressão múltipla de Cox segundo a modelagem hierarquizada, considerando os blocos de variáveis. Com base no critério de seleção de variáveis, de cada bloco foram excluídas as seguintes: escolaridade do chefe da família (B1); perdas fetais anteriores e intervalo interpartal (B2); presença de maus tratos na gestação (B3); hospitalização durante a gestação (B4); tipo do hospital (B5); RCIU (B6); presença de sofrimento fetal (B7).

A única variável socioeconômica que permaneceu no modelo final foi o local de residência da mãe. Ao adicionar as variáveis sobre história reprodutiva materna, histórico anterior de aborto provocado e de cesariana, estas se mostraram associadas à mortalidade neonatal. Das condições psicossociais, somente os nascimentos originados de mães adolescentes permaneceram no modelo. Quando acrescentadas as variáveis sobre as condições da gestação atual (B4), sangramento e adequação de pré-natal foram para o modelo final. Ao se adicionarem as variáveis 
Figura 1

Sobrevida segundo categorias de peso ao nascer da coorte de recém-nascidos de muito baixo peso. Região Sul do Município de São Paulo, Brasil, 2000/2001.

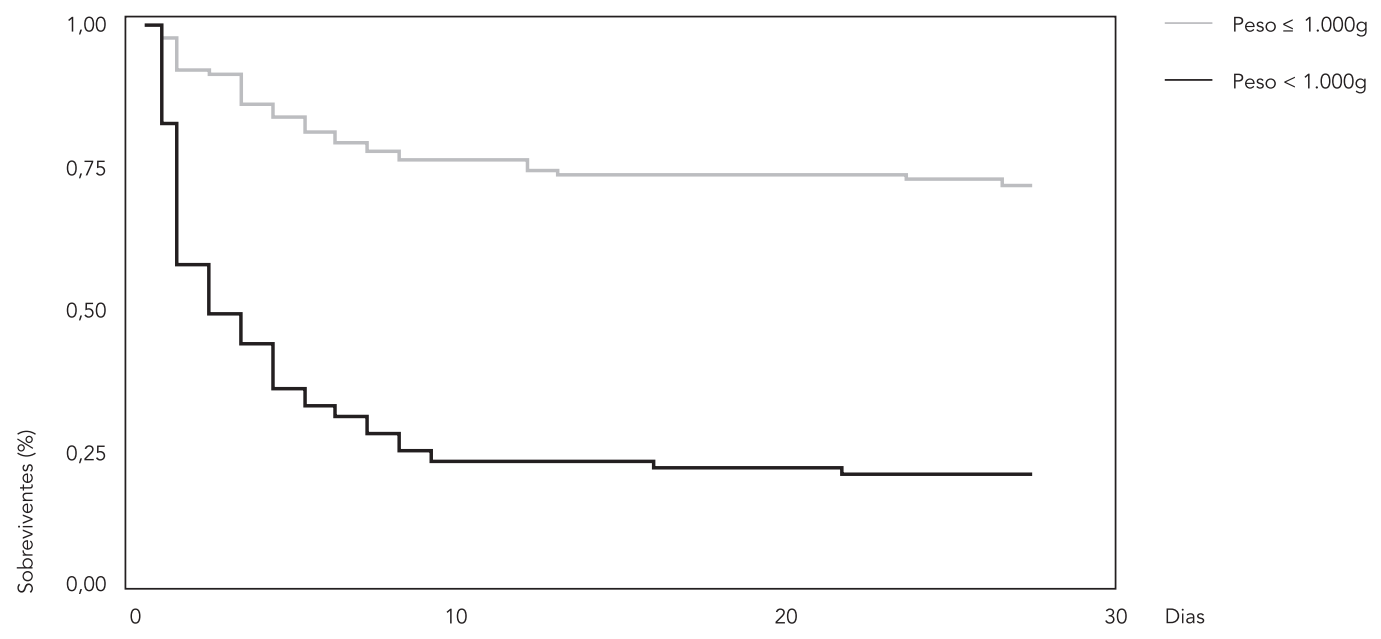

Figura 2

Sobrevida segundo categorias de idade gestacional da coorte de recém-nascidos de muito baixo peso. Região Sul do Município de São Paulo, Brasil, 2000/2001.

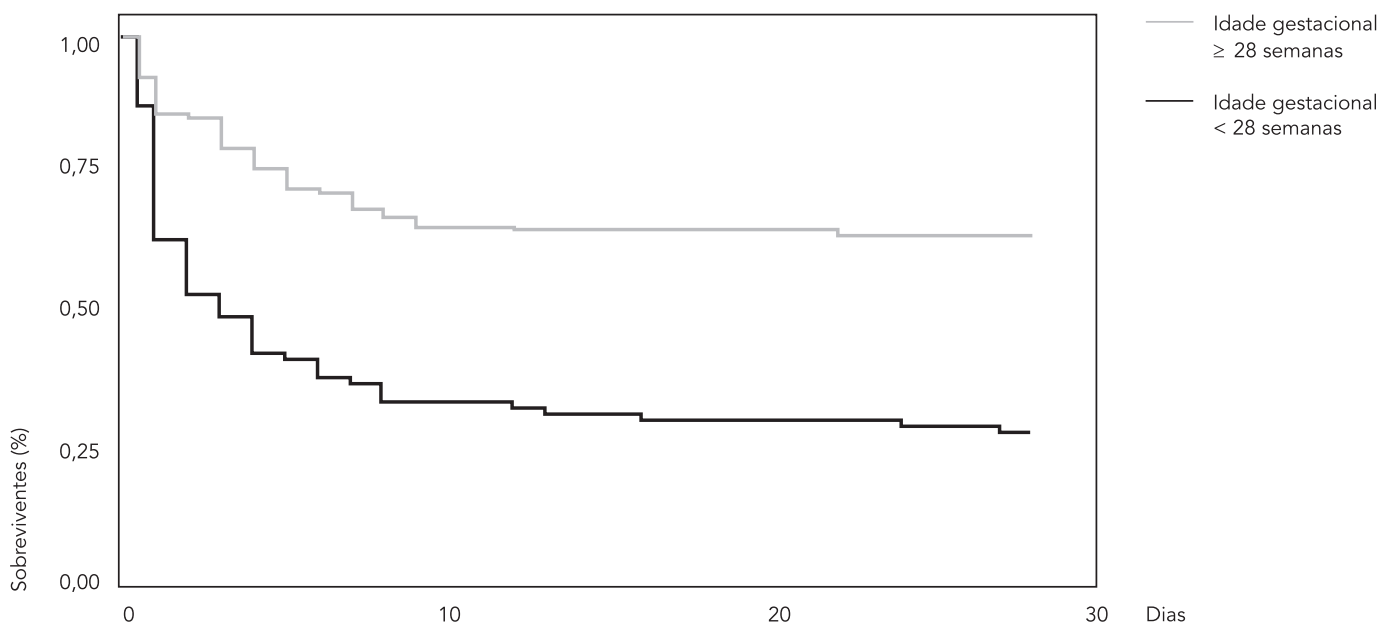


sobre condições do parto (B5), apenas o tipo de parto ficou no modelo. Quando se acrescentaram as variáveis sobre características do recémnascido (B6), permaneceram no modelo o peso ao nascer e o índice de Apgar. Por último, ao se acrescentarem as variáveis relativas aos cuidados com o recém-nascido, apenas a variável berçário normal permaneceu no modelo (Tabela 1). As demais variáveis foram excluídas nos passos anteriores da modelagem.

Fatores de risco na coorte de recém-nascidos de muito baixo peso. Região Sul do Município de São Paulo, Brasil, $2000 / 2001$.

\begin{tabular}{|c|c|c|c|c|c|c|c|c|}
\hline Variáveis & Óbitos & $\%$ & Sobreviventes & $\%$ & HR bruta & $\begin{array}{c}\text { HR } \\
\text { ajustada }\end{array}$ & IC95\% & Valor de $\mathrm{p}$ \\
\hline \multicolumn{9}{|c|}{ Bloco 1 - Características socioeconômicas das } \\
\hline \multicolumn{9}{|l|}{ mães e das famílias } \\
\hline Local de residência da mãe & & & & & & & & 0,01 \\
\hline Não favela & 84 & 48,3 & 90 & 51,7 & 1,00 & 1,00 & & \\
\hline Favela & 28 & 71,8 & 11 & 28,2 & 1,71 & 1,76 & $1,14-2,70$ & \\
\hline \multicolumn{9}{|c|}{ Bloco 2 - História reprodutiva materna } \\
\hline Parto cesariano anterior & & & & & & & & 0,02 \\
\hline Não * & 81 & 47,9 & 88 & 52,1 & 1,00 & 1,00 & & \\
\hline Sim & 31 & 70,4 & 13 & 29,5 & 1,79 & 1,67 & $1,10-2,55$ & \\
\hline Aborto provocado anterior & & & & & & & & $<0,01$ \\
\hline Não * & 107 & 51,4 & 101 & 48,6 & 1,00 & 1,00 & & \\
\hline Sim & 5 & 100,0 & 0 & 0,0 & 4,29 & 3,73 & $1,47-9,43$ & \\
\hline \multicolumn{9}{|c|}{ Bloco 3 - Características psicossociais das mães } \\
\hline Mãe adolescente (<20 anos) & & & & & & & & 0,03 \\
\hline Sim & 73 & 48,7 & 77 & 51,3 & 1,00 & 1,00 & & \\
\hline Não & 39 & 61,9 & 24 & 38,1 & 1,34 & 1,56 & $1,04-2,36$ & \\
\hline \multicolumn{9}{|c|}{ Bloco 4 - Características da gestação atual } \\
\hline Sangramento & & & & & & & & 0,02 \\
\hline Não & 90 & 49,7 & 91 & 50,3 & 1,00 & 1,00 & & \\
\hline $\operatorname{Sim}$ & 22 & 68,7 & 10 & 31,2 & 1,62 & 1,79 & $1,11-2,89$ & \\
\hline Adequação pré-natal & & & & & & & & $<0,01$ \\
\hline Adequado & 57 & 47,1 & 64 & 52,9 & 1,00 & 1,00 & & \\
\hline Inadequado & 30 & 50,0 & 30 & 50,0 & 1,14 & 1,10 & $0,70-1,72$ & \\
\hline Não fez & 25 & 78,1 & 7 & 21,9 & 2,05 & 2,04 & $1,26-3,28$ & \\
\hline \multicolumn{9}{|l|}{ Bloco 5 - Características do parto } \\
\hline Tipo de parto & & & & & & & & $<0,01$ \\
\hline Normal & 88 & 63,3 & 51 & 36,7 & 1,00 & 1,00 & & \\
\hline Cesárea & 24 & 32,4 & 50 & 67,6 & 0,40 & 0,44 & $0,27-0,69$ & \\
\hline \multicolumn{9}{|c|}{ Bloco 6 - Características do recém nascido } \\
\hline Peso ao nascer (g) & & & & & & & & $<0,01$ \\
\hline$\geq 1.000$ & 33 & 28,7 & 82 & 71,3 & 1,00 & 1,00 & & \\
\hline$<1.000$ & 79 & 80,6 & 19 & 19,4 & 4,77 & 4,52 & $2,95-6,94$ & \\
\hline Índice de Apgar & & & & & & & & $<0,01$ \\
\hline $7-10$ & 16 & 28,1 & 41 & 71,9 & 1,00 & 1,00 & & \\
\hline $0-6$ & 78 & 62,4 & 47 & 37,6 & 3,06 & 2,21 & $1,28-3,83$ & \\
\hline Ignorado & 18 & 58,1 & 13 & 42 & 2,50 & 1,70 & $0,85-3,41$ & \\
\hline \multicolumn{9}{|c|}{ Bloco 7 - Cuidados com o recém nascido } \\
\hline Berçário normal & & & & & & & & 0,03 \\
\hline Não & 105 & 57,9 & 78 & 42,6 & 1,00 & 1,00 & & \\
\hline Sim & 7 & 23,3 & 23 & 76,7 & 0,33 & 0,42 & $0,19-0,92$ & \\
\hline
\end{tabular}

* Inclui 90 mães primíparas: 43 óbitos e 47 sobreviventes. 


\section{Discussão}

A comparação de resultados sobre a mortalidade em coortes de MBPN não é uma tarefa fácil, pois há diferenças na composição das diversas coortes. Algumas excluem nascimentos com idade gestacional abaixo de 24 semanas 3 ou de 22 semanas 17,18; outras excluem nascimentos com

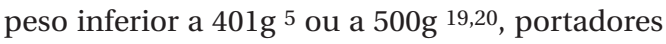
de malformação congênita 6,8 e gemelares 21 , os quais apresentam elevado risco de morte.

As perdas foram elevadas, principalmente, em razão da dificuldade de localização dos endereços registrados nas declarações de nascido vivo, a base de dados empregada para a obtenção da coorte. Em uma grande metrópole, essa dificuldade se deve à existência de áreas de loteamentos clandestinos e de ocupação ilegal 22, cujos residentes frequentemente informam endereços oficiais que se situam na margem dessas ocupações. Por outro lado, ocorreram perdas de localização de prontuários em virtude da recusa de participação de dois hospitais privados que concentram atendimento da população de alta renda. De certa forma, essas perdas se compensaram, não resultando em diferença significativa entre o peso médio das perdas e o da população de estudo; no entanto, as perdas levaram à superestimação da mortalidade. Parte da elevada probabilidade de morte encontrada na coorte também pode ser creditada à definição da população de estudo, que seguiu a CID-10, incluindo todos os nascidos vivos que apresentavam sinais vitais, e nem todos os recém-nascidos foram atendidos em hospitais terciários.

A probabilidade de morte se aproxima da encontrada em um estudo 20 realizado em hospitais da Região Nordeste do Brasil em 2002/2003, cujos resultados foram mais elevados que os de estudos realizados em UTIN das regiões Sudeste e Sul 6,19,20. Essa diferença pode ser creditada à superestimação da mortalidade, à composição da coorte (inclusão de todos os recém-nascidos com sinais vitais) e ao perfil da assistência aos recém-nascidos dos demais estudos. Tanto os nossos resultados como os obtidos em outros estudos nacionais mostram que a mortalidade observada era mais elevada que a obtida em estudos internacionais, o que pode ser creditado à qualidade da atenção disponível nas UTIN 19,20.

Houve concentração de ocorrência de óbitos até o quarto dia de vida; posteriormente observou-se um lento declínio, padrão semelhante ao encontrado em países desenvolvidos 9,23.

A elevada mortalidade no primeiro dia de vida e na sala de parto, juntamente com a elevada mortalidade dos recém-nascidos com peso inferior a $1.000 \mathrm{~g}$, de prematuros extremos e a ausên- cia de sobreviventes com peso inferior a $700 \mathrm{~g}$, sugere que condutas mais ativas destinaram-se a recém-nascidos de maior viabilidade. Esse resultado condiz com o baixo emprego de surfactante observado nos nascimentos com peso inferior a $1.000 \mathrm{~g}$. Alguns países europeus reservam condutas mais pró-ativas aos recém-nascidos de maior viabilidade 2,18,24,25, porém cabe comentar que, nesses locais, há parâmetros definidos para o emprego de tais condutas e há participação de familiares no processo de definição desses parâmetros 26,27.

A elevada mortalidade de recém-nascidos de maior viabilidade (peso acima de $1.000 \mathrm{~g}$ e idade gestacional de 28 semanas e mais) pode estar, em parte, refletindo o acesso diferenciado aos cuidados intensivos e a qualidade destes.

A elevada probabilidade de morte da coorte pode também decorrer da complexa rede de fatores de risco encontrada. À semelhança de outros estudos nacionais 7,28 , a variável residência localizada em favelas associou-se à mortalidade neonatal. Estudo realizado no Rio de Janeiro encontrou associação entre a residência materna e moradias precárias, de um lado, e nascimentos de baixo peso e pré-termo ${ }^{29}$, de outro. Porém, a precariedade das habitações e a elevada aglomeração intradomiciliar não exercem influência direta sobre a mortalidade neonatal. Essa variável expressa aspectos contextuais que vão além desses fatores, como o efeito da pobreza e das situações geradoras de estresse em virtude da presença da violência e de dificuldades de acesso a serviços públicos, entre os quais os de saúde 30 da população residente nessas áreas. Assim, residência em favela trata-se de uma variável contextual que expressa a vulnerabilidade social e a heterogeneidade do tecido social metropolitano 22.

Neste estudo, a variável mãe adolescente foi incluída no bloco condições psicossocial das mães, pois se considerou que a gestação na adolescência pode constituir fator de estresse para mães e famílias 12,31. Contudo, esse fator tem apresentado resultados divergentes, mesmo quando ajustados por outras variáveis. Alguns estudos não encontraram associação entre gestação na adolescência e óbitos neonatais 28,32 , enquanto outros a identificaram como fator de risco 2,9,33. Possivelmente, distintas realidades sociais ou diferentes tipos de análise possam estar produzindo esses resultados.

No modelo final, entre as variáveis que expressam a história reprodutiva materna, parto cesariano e aborto provocado anteriores associaram-se ao desfecho. O parto cesariano prévio pode indicar a presença de fatores maternos negativos em gestações anteriores (problemas placentários e/ou anomalias cérvico-uterinas), 
os quais estão associados a nascimentos de prétermo e que podem ter se repetido na gestação atual, contribuindo para a ocorrência de óbitos neonatais ${ }^{34}$. O aborto provocado prévio foi identificado como fator de risco para nascimentos de pré-termo 34,35 e para óbitos neonatais precoces 33 . Esse efeito é observado principalmente nos abortos realizados por curetagem, cujas sequelas são frequentes (insuficiência placentária, endometrite, infecções pélvicas e laceração cervical) 35 . Como o aborto provocado é ilegal no Brasil, a curetagem e outros métodos autoinfligidos mais agressivos são comuns na população de menor poder aquisitivo 36 .

Com relação às características da gestação atual, a presença de sangramento vaginal e a adequação de assistência pré-natal mostraramse associadas à ocorrência de óbitos neonatais. $\mathrm{O}$ sangramento vaginal pode ser um sinal de ruptura prematura de membranas ou de trabalho de parto prematuro; outra hipótese é a sua associação com infecções uterinas inaparentes, as quais também estão associadas ao parto prematuro 7 . Além do sangramento, a hipertensão tem sido considerada fator de risco para óbito neonatal, como já mostrado em estudos com recém-nascidos internados em UTIN 6. Nesta investigação, apenas a eclâmpsia associou-se aos óbitos neonatais; no processo de modelagem, essa afecção foi retirada do modelo ao se acrescentarem as variáveis mais proximais ao desfecho, sugerindo que esta pode se encontrar no caminho causal dos nascimentos MBPN.

A assistência pré-natal ausente ou inadequada vem sendo apontada em diversos estudos como fator de risco para a mortalidade neonatal $28,33,34$. Seu efeito sobre a mortalidade neonatal é indireto, uma vez que a adequada assistência pré-natal poderia identifica algum problema e atuar a tempo. Isso reduziria o impacto de possíveis intercorrências da gestação, prevenindo, desse modo, nascimentos MBPN e óbitos neonatais.

A realização de partos cesarianos mostrou efeito protetor para a mortalidade desses neonatos, resultado compatível com os de vários estudos nacionais sobre mortalidade neonatal realizados em recém-nascidos internados em UTINs 6,32. Resultados semelhantes foram obtidos em estudos internacionais 37,38 .

Com relação às características dos recémnascidos, após os ajustes, permaneceram no modelo os nascimentos com peso abaixo de $1.000 \mathrm{~g}$ e aqueles com índice de Apgar menor que 7 no quinto minuto. A idade gestacional foi excluída do modelo possivelmente porque sua estimação foi menos precisa que o peso ao nascer. Esses resultados são concordantes entre si: o baixo índice de Apgar no quinto minuto representa a baixa vitalidade dos recém-nascidos, estabelecida por meio de medidas clínicas; por outro lado, o extremo baixo peso pode estar representando um parâmetro epidemiológico de sobrevivência desses recém-nascidos. Outros estudos identificaram baixos valores do índice de Apgar como risco para a mortalidade neonatal em UTIN para recém-nascidos de baixo peso ou muito baixo peso 6,32. Resultados semelhantes foram encontrados em uma coorte de recém-nascidos com menos de $1.500 \mathrm{~g}$ em várias UTIN das regiões Sul e Sudeste 6 .

Neste estudo, o emprego ou não de determinados procedimentos clínicos foi analisado apenas como potencial de resolubilidade da assistência ${ }^{39}$. Os recém-nascidos da coorte internados em berçário normal apresentavam menor chance de ocorrência de óbito neonatal, sugerindo que estes tinham maiores chances de sobrevivência, não necessitando de cuidados intensivos. A internação em UTIN também havia mostrado efeito protetor na análise múltipla do bloco de atenção ao recém-nascido, mas esta variável foi retirada do modelo quando foi ajustada pela presença das demais variáveis.

O presente estudo é retrospectivo e pode estar sujeito a vieses de memória das mães e de aferição na coleta de dados. Além disso, o poder desta pesquisa pode ter sido insuficiente para avaliar o efeito de algumas variáveis. Outra limitação está relacionada à obtenção da coorte. Nesta, o risco de morte neonatal encontra-se superestimado, pois as perdas foram mais elevadas entre sobreviventes que entre os óbitos, o que também pode ter superestimado medidas de associação de algumas covariáveis. As informações sobre os cuidados prestados no parto e ao recém-nascido são mais frágeis neste tipo de estudo do que naqueles de base hospitalar, uma vez que não são passíveis de padronização prévia e podem ocorrer omissões de registro.

Ocorreram mudanças sociais e na oferta de serviços de saúde ao longo do tempo, resultando no declínio da mortalidade neonatal no Município deSão Paulo, onde esta passou de 10,3 para 8,1 por mil nascidos vivos em 2009. Comportamento semelhante foi observado na Região Sul, onde a mortalidade neonatal passou de 10,7 em 2001 para 8,0 por mil nascidos vivos em 2009. Essa semelhança se deve, em parte, ao fato de a Região Sul concentrar cerca de $26 \%$ dos nascimentos do Município de São Paulo em 2001 e 2009 (Fundação Seade. http://www.seade.gov.br/produtos/ mortinf/tabelas/2009/pdf/tabela05_2009_distri tos.pdf, acessado em 06/Abr/2011; http://www. seade.gov.br/produtos/anuario/mostra_tabela. php? anos $=2002 \&$ tema $=$ dem $\&$ tabpesq $=\operatorname{dem} 20$ 
02_57\&tabela=null, acessado em 06/Abr/2011; http://www.seade.gov.br/produtos/anuario/ mostra_tabela.php?anos $=2002 \&$ tema $=$ dem $\&$ ta bpesq=dem2001_21\&tabela=null, acessado em 06/Abr/2011).

É possível que alguns fatores de risco tenham se modificado entre a realização do estudo e os dias de hoje, tendo em vista que ocorreram melhorias na assistência à gestante e ao parto no país 40,41. No período de 2003 a 2009, houve redução de $15,1 \%$ para $13,6 \%$ da proporção de mães adolescentes, e crescimento de $10 \%$ de mães que tiveram sete ou mais consultas de pré-natal no Município de São Paulo (Secretaria Municipal de Saúde da Prefeitura do Município de São Paulo. SINASC Tabnet. http://ww2.prefeitura.sp.gov.br/ cgi/deftohtm.exe?secretarias/saude/TABNET/ SMS/nasc_sp.def, acessado em 06/Abr/2011). Ocorreram também outras iniciativas, como o programa mãe paulistana 42 , que podem ter contribuído para o acesso mais imediato e referenciado para as gestações de risco. Os dados mostram a expansão da assistência pré-natal, porém é preciso lembrar que sua utilização tem se mostrado heterogênea no Município de São Paulo, decrescendo com o aumento da vulnerabilidade social 43 .

Com relação às condições de vida verificouse crescimento da escolaridade. Em 2003, a proporção de nascidos vivos de mães de baixa escolaridade era de $7,6 \%$ na Região Sul e de 5,9\% no
Município de São Paulo. Em 2009, essa proporção era, respectivamente, de 2,9\% e de 2,3\% (Secretaria Municipal de Saúde da Prefeitura do Município de São Paulo. SINASC Tabnet. http://ww2.pre feitura.sp.gov.br/cgi/deftohtm.exe?secretarias/ saude/TABNET/SMS/nasc_sp.def, acessado em 06/Abr/2011). Todavia, esse crescimento não se traduz necessariamente na redução de proporção da população vivendo em favelas, já que alguns estudos têm mostrado que houve aumento da escolaridade nesse grupo 22,44.

\section{Conclusões}

O presente estudo encontrou mortalidade elevada, tanto para os nascimentos de extremo baixo peso quanto para os de maior viabilidade, indicando que é possível obter ganhos com a melhor identificação de gestações de risco e com os cuidados prestados a esse grupo de recémnascidos. Estudos populacionais podem explicitar a contribuição para desfechos negativos da vulnerabilidade social e de fatores de risco, como residência em favelas, mães adolescentes e/ou com história prévia de aborto inseguro, os quais podem estar ausentes em estudos realizados em hospitais terciários. Portanto, a ampliação de medidas de prevenção de nascimentos de prétermo pode contribuir para a redução dos atuais níveis de mortalidade.

\section{Resumo}

Estudos populacionais sobre mortalidade neonatal de nascimentos de muito baixo peso ao nascer contribuem para identificar sua complexa rede de fatores de risco. Foi estudada uma coorte de 213 recém-nascidos com peso inferior a 1.500g (112 óbitos neonatais e 101 sobreviventes) na Região Sul do Município de São Paulo, Brasil, em 2000/2001. Foram realizadas entrevistas domiciliares e obtidos dados de prontuários hospitalares. Foi realizada análise de sobrevida e empregada regressão múltipla de Cox. A elevada mortalidade na sala de parto, no primeiro dia de vida e ausência de sobreviventes $<700 \mathrm{~g}$ dos nascimentos $<1.000 \mathrm{~g}$ e com menos de 28 semanas sugere que condutas mais ativas destinam-se a nascituros de maior viabilidade. Mães residentes em favela, com história anterior de cesárea e aborto provocado, adolescentes, com sangramento vaginal e ausência de pré-natal aumentaram o risco de óbito neonatal. Partos cesarianos e internação em berçários mostraram efeito protetor. O peso ao nascer abaixo de 1.000g e Apgar menor que 7 foram risco. A elevada mortalidade está associada às condições de vida, características maternas e dos nascimentos e condições assistenciais. A melhoria da atenção pré-natal e ao recém-nascido pode atuar na redução da mortalidade.

Recém-Nascido de Baixo Peso; Mortalidade Neonatal; Estudos de Coortes; Análise de Sobrevida 


\section{Colaboradores}

M. F. Almeida, G. P. Alencar e D. Schoeps contribuíram com o desenho do estudo, coleta de dados, análise e redação do artigo. H. M. D. Novaes contribuiu com o desenho do estudo, coleta de dados e redação do artigo. O. Campbell e L. C. Rodrigues contribuíram com a análise de dados e redação do artigo.

\section{Referências}

1. Martines J, Paul VK, Bhutta ZA, Koblinsky M, Soucat A, Walker N, et al. Neonatal survival: a call for action Published online. Lancet 2005; 365:1189-97.

2. Ravelli ACJ, Tromp M, van Huis M, Steegers EAP, Tamminga P, Eskes M, et al. Decreasing perinatal mortality in the Netherlands, 2000-2006: a record linkage study. J Epidemiol Community Health 2009; 63:761-5.

3. Draper ES, Zeitlin J, Fenton AC, Weber T, Gerrits $\mathrm{J}$, Martens $\mathrm{G}$, et al. Investigating the variations in survival rates for very preterm infants in 10 European regions: the MOSAIC birth cohort. Arch Dis Child Fetal Neonatal Ed 2009; 94:F158-63.

4. Murphy BP, Armstrong K, Ryan CA, Jenkins JG. Benchmarking care for very low birthweight infants in Ireland and Northern Ireland. Arch Dis Child Fetal Neonatal Ed 2010; 95:F30-5.

5. Duarte JLMB, Mendonça GAS. Fatores associados à morte neonatal em recém-nascidos de muito baixo peso em quatro maternidades no Município do Rio de Janeiro, Brasil. Cad Saúde Pública 2005; 21:181-91.

\section{Agradecimentos}

Ao Conselho Nacional de Desenvolvimento Científico e Tecnológico (CNPq-403335/2004).
6. Almeida MFB, Guinsburg R, Martinez FER, Procianoy RS, Leone CR, Marba STM, et al. Fatores perinatais associados ao óbito precoce em prematuros nascidos nos centros da Rede Brasileira de Pesquisas Neonatais. J Pediatr (Rio J.) 2008; 84:300-7.

7. Silva AMR, Almeida MF, Matsuo T, Soares DA. Fatores de risco para nascimentos pré-termo em Londrina, Paraná, Brasil. Cad Saúde Pública 2009; 25:2125-38.

8. Duarte JLMB, Mendonça GAS. Comparação da mortalidade neonatal em recém-nascidos de muito baixo peso ao nascimento, em maternidades do Município do Rio de Janeiro, Brasil. Cad Saúde Pública 2005; 21:1441-7.

9. Bacak SJ, Baptiste-Roberts K, Amon E, Ireland B, Leet $\mathrm{T}$. Risk factors for neonatal mortality among extremely-low-birth-weight infants. Am J Obstet Gynecol 2005; 192:862-7.

10. Organização Mundial da Saúde. Classificação estatística internacional de doenças e problemas relacionados à saúde. 10a revisão. v. 2. São Paulo: Edusp; 1994. 
11. Reichenheim ME, Moraes CL, Hasselmann MH. Equivalência semântica da versão em português do instrumento Abuse Assessment Screen para rastrear a violência contra a mulher grávida. Rev Saúde Pública 2000; 34:610-6.

12. Schoeps D, Almeida MF, Alencar GP, França Jr. I, Novaes HMD, Siqueira AAF, et al. Fatores de risco para mortalidade neonatal precoce. Rev Saúde Pública 2007; 41:1013-22.

13. Almeida MF, Novaes HMD, Alencar GP, Rodrigues LC. Neonatal mortality: socio-economic, health services risk factors and birth weight in the City of São Paulo. Rev Bras Epidemiol 2002; 5:93-107.

14. Williams RL, Creasy RK, Cunningham GC, Hawes WE, Norris FD, Tashiro M. Fetal growth and perinatal viability in California. Obstet Gynecol 1982; 59:624-32.

15. Szklo M, Nieto FJ. Measuring disease occurrence. In: Szklo M, Nieto FJ, editors. Epidemiology. Beyond the basics. Gaithersburg: Aspen Publication; 2000. p. 55-90.

16. Victora CG, Huttly SR, Fuchs SC, Olinto MTA. The role of conceptual frameworks in epidemiological analysis: a hierarchical approach. Int J Epidemiol 1997; 26:224-7.

17. Costa MCN, Mota ELA, Paim JS, Silva LMV, Teixeira MG, Mendes CMC. Mortalidade infantil no Brasil em períodos recentes de crise econômica. Rev Saúde Pública 2003; 37:699-706.

18. Tommiska V, Heinonen K, Lehtonen L, Renlund M, Saarela T, Tammela O, et al. No improvement in outcome of nationwide extremely low birth weight infant populations between 1996-1997 and 19992000. Pediatrics 2007; 119:29-36.

19. Kramer MS, Barros FC, Demissie K, Liu S, Kiely J, Joseph KS. Does reducing infant mortality depend on preventing low birthweight? An analysis of temporal trends in the Americas. Paediatr Perinat Epidemiol 2005; 19:445-51.

20. Castro EMC, Leite AJM. Mortalidade hospitalar dos recém-nascidos com peso de nascimento menor ou igual a 1.500g no município de Fortaleza. J Pediatr (Rio J.) 2007; 83:27-32.

21. Ortiz LP, Camargo A. Mortalidade infantil no estado em 2003: níveis diminuem e diferenciais regionais persistem. SP Demográfico 2004; 5(10).

22. Gonçalves R, Saraiva C. Vulnerabilidade social, segregação urbana e desigualdades intraregionais (Salvador, Rio de Janeiro e São Paulo). http://www.centrodametropole.org.br/index. php?section=content\&subsection_id=5\&content_ id=548 (acessado em 03/Abr/2011).

23. Meadow W, Lee G, Lin K, Lantos J. Changes in Mortality low birth weight infants in 1990s: implications for treatment decisions and resource use. Pediatrics 2004; 113:1223-9.

24. de Kleine MJK, den Ouden AL, Kollée LAA, Ilsen A, van Wassenaer AG, Brand R, et al. Lower mortality but higher neonatal morbidity over a decade in very preterm infants. Paediatr Perinat Epidemiol 2007; 21:15-25.

25. Zeitlin J, Draper ES, Kollée L, Milligan D, Boerch $\mathrm{K}$, Agostino R, et al. Differences in rates and shortterm outcome of live births before 32 weeks of gestation in Europe in 2003: results from the MOSAIC cohort. Pediatrics 2008; 121:936-44.
26. Marlow N. Outcome following extremely preterm birth. Current Pediatrics 2004; 14:275-83.

27. Janvier A, Barrington K. The ethics of neonatal resuscitation at the margins of viability: informed consent and outcomes. J Pediatr 2005; 147:579-85.

28. Almeida SDM, Barros MBA. Atenção à saúde e mortalidade neonatal: estudo caso-controle realizado em Campinas, SP. Rev Bras Epidemiol 2004; 7:22-34.

29. Vettore MV, Gama SG, Lamarca GA, Schilithz AO, Leal MC. Housing conditions as a social determinant of low birthweight and preterm low birthweight. Rev Saúde Pública 2010; 44:1021-31.

30. Rodrigues RI. Moradia precária e violência na cidade de São Paulo. Rio de Janeiro: Instituto de Pesquisa Econômica Aplicada; 2006. (Texto para Discussão, 1187).

31. Misra DP, O'Campo P, Strobino D. Testing a sociomedical model from preterm delivery. Paediatr Perinat Epidemiol 2001; 15:110-22.

32. Ribeiro AM, Guimarães MJ, Lima MC, Sarinho SW, Coutinho SB. Fatores de risco para mortalidade neonatal em crianças com baixo peso ao nascer. Rev Saúde Pública 2009; 43:246-55.

33. Araújo BF, Bozzetti MC, Tanaka ACA. Early neonatal mortality in Caxias do Sul: a cohort study. J Pediatr (Rio J.) 2000; 76:200-6.

34. Allen MC, Alexander GR, Tompkins ME, Hulsey TC. Racial differences in temporal changes in newborn viability and survival by gestational age. Paediatr Perinat Epidemiol 2000: 14:152-8.

35. Bracken MB. Induced abortion as a risk factor for perinatal complications: a review. Yale J Biol Med 1978; 51:539-48.

36. Menezes G, Aquino EML. Pesquisa sobre o aborto no Brasil: avanços e desafios para o campo da saúde coletiva. Cad Saúde Pública 2009; 25 Suppl 2:S193-204.

37. Muhuri PK, Macdorman MF, Menacker R. Method of delivery and neonatal mortality among very low birth weight infants in the United States. Matern Child Health J 2006; 10:47-53.

38. Malloy MH. Impact of cesarean section on neonatal mortality rates among very preterm infants, in the United States, 2000-2003. Pediatrics 2008; 122:285-92.

39. Novaes MHD. Mortalidade neonatal e avaliação da qualidade de atenção ao parto e ao recém-nascido no Município de São Paulo [Tese de Livre-Docência]. São Paulo: Faculdade de Medicina, Universidade de São Paulo; 1999.

40. Barros FC, Matijasevich A, Requejo JH, Giugliani E, Maranhão AG, Monteiro CA, et al. Recent trends in maternal, newborn, and child health in Brazil: progress toward Millennium Development Goals 4 and 5. Am J Public Health 2010; 100:1877-89.

41. Malta DC, Duarte EC, Escalante JJC, Almeida MF, Sardinha LMV, Macário EM, et al. Mortes evitáveis em menores de um ano, Brasil, 1997 a 2006: contribuições para a avaliação de desempenho do Sistema Ưnico de Saúde. Cad Saúde Pública 2010; 26:481-91. 
42. Secretaria Municipal de Saúde de São Paulo. Decreto municipal $\mathrm{n}^{\circ}$. 46.966 , de 02 de fevereiro de 2006. Regulamenta a lei $n^{\circ}$. 13.211 , de 13 de novembro de 2001, estruturando a Rede de Proteção à Mãe Paulistana, para a gestão e execução da rede de serviços de saúde de assistência obstétrica e neonatal no Município de São Paulo. Diário Oficial do Município, São Paulo 2006; 3 fev.

43. Minuci EG, Almeida MF. Diferenciais intra-urbanos de peso ao nascer no município de São Paulo. Rev Saúde Pública 2009; 43:256-66.
44. Pasternak S. Espaço e população nas favelas de São Paulo. In: Anais do XIII Encontro da Associação Brasileira de Estudos Populacionais. http://www. abep.nepo.unicamp.br/docs/anais/pdf/2002/GT_ MA_ST21_Pasternak_texto.pdf (acessado em 06/ Abr/2011).

Recebido em 08/Dez/2010

Versão final reapresentada em 06/Abr/2011

Aprovado em 18/Abr/2011 